\title{
Cytokine mRNA profiling identifies $B$ cells as a major source of RANKL in rheumatoid arthritis
}

\author{
Lorraine Yeo, ${ }^{1}$ Kai-Michael Toellner, ${ }^{1}$ Mike Salmon, ${ }^{1}$ Andrew Filer, ${ }^{1,2}$ \\ Christopher D Buckley, ${ }^{1,2}$ Karim Raza, ${ }^{1,2}$ Dagmar Scheel-Toellner ${ }^{1}$
}

- Supplementary figures are published online only. To view these files please visit the journal online (http://ard.bmj. com)

${ }^{1}$ MRC Centre for Immune Regulation, Institute for Biomedical Research, College of Medical and Dental Sciences, University of Birmingham, Birmingham, UK

${ }^{2}$ Sandwell and West Birmingham Hospitals NHS Trust, Birmingham, UK

\section{Correspondence to} Dr Dagmar Scheel-Toellner, Rheumatology Research Group MRC Centre for Immune Regulation, School of Immunity and Infection, College of Medical and Dental Sciences, University of Birmingham, Birmingham B15 2T, UK: d.scheel@bham.ac.uk

Accepted 13 June 2011 Published Online First 8 July 2011

\section{UNLOCKID}

This paper is freely available online under the BMJ Journals unlocked scheme, see http:// ard.bmj.com/info/unlocked.dtl

\begin{abstract}
Objectives In rheumatoid arthritis (RA), a complex cytokine network drives chronic inflammation and joint destruction. So far, few attempts have been made to identify the cellular sources of individual cytokines systematically. Therefore, the primary objective of this study was systematically to assess the cytokine messenger RNA expression profiles in the five largest cell populations in the synovial fluid and peripheral blood of RA patients. To reflect the in vivo situation as closely as possible, the cells were neither cultured nor stimulated ex vivo.
\end{abstract}

Methods Inflammatory cells from 12 RA patients were sorted into CD4 and CD8 T cells, B cells, macrophages and neutrophils. mRNA expression for 41 cytokines was determined by real-time PCR using microfluidic cards. Receptor activator nuclear factor kappa B ligand (RANKL) (TNFSF11) expression by B cells was further confirmed by flow cytometry and by immunofluorescence staining of frozen sections of synovial tissue from patients with RA.

Results The detection of cytokines characteristic for $T$ cells and myeloid cells in the expected populations validated this methodology. Beyond the expected cytokine patterns, novel observations were made. Striking among these was the high expression of mRNA for RANKL in B cells from synovial fluid. This observation was validated at the protein level in synovial tissue and fluid.

Conclusions RANKL, the key cytokine driving bone destruction by osteoclast activation, is produced by synovial $B$ cells in RA. This observation is of importance for our understanding of the role of $B$ cells in RA and their therapeutic targeting.

Cytokines are recognised as important factors in the pathogenesis of rheumatoid arthritis (RA). ${ }^{1} 2$ In RA, the joint is infiltrated by multiple inflammatory cell populations including $\mathrm{T}$ cells, B cells, macrophages and neutrophils, all of which contribute to the local cytokine network. Elucidating the cytokine network in RA represents an important challenge for furthering our understanding of disease pathogenesis. ${ }^{3-6}$ The first step towards achieving this goal is to determine the cellular sources of the cytokines found in the rheumatoid joint.

Early studies of T-cell cytokine production in RA indicated that synovial T cells produce low levels of interleukin (IL) 2 and interferon gamma (IFNy). ${ }^{7-9}$ More recently, IL-17-producing T cells have been reported in the RA synovium, where they are thought to promote inflammation by activating local stromal cells. ${ }^{10}{ }^{11}$ Furthermore, RA synovial $T$ cells have been shown to produce pro-inflammatory cytokines such as IL-6, tumour necrosis factor (TNF) alpha and TNF family members receptor activator nuclear factor kappa B ligand (RANKL) and lymphotoxin beta (LT- $\beta$ ). ${ }^{12-15} \mathrm{~B}$ cells have proved to be a successful therapeutic target in RA, highlighting an important role for this cell type beyond their ability to produce antibody. RA synovial B cells can produce several cytokines including LT- $\beta$, which may be required for synovial ectopic lymphoid tissue formation and IL-6, which implicates a direct pro-inflammatory role. ${ }^{16} 17$ On the other hand, an IL-10-producing B-cell subset has been attributed with anti-inflammatory properties. ${ }^{18} 19$ So far, this population has not been described in the RA synovium. Macrophages are regarded as an important source of pro-inflammatory cytokines found in the RA synovium including TNF $\alpha$, IL-1 and IL-18. ${ }^{13} 2021$ Through expression of IL-7, IFN $\beta$, IL-23, B-cell-activating factor of the TNF family (BAFF) and (a proliferation-inducing ligand) APRIL, synovial macrophages are thought to play a role in synovial T-cell and B-cell differentiation and survival. ${ }^{22-26}$ Neutrophils represent the largest cell population in rheumatoid synovial fluid and produce a range of cytokines including IL-1 $\beta$, BAFF, APRIL and transforming growth factor beta (TGFB) as well as the chemokines CCL3 and CXCL8. ${ }^{25}$ 27-30

To date, no study has systematically determined cytokine production by synovial fluid cell populations. The aim of the present study was therefore to identify the predominant cellular sources of cytokines in RA by comparing their expression across five populations in RA synovial fluid and peripheral blood. To achieve this we developed a new approach, which has the advantage of characterising cytokine profiles in cells directly ex vivo without culture or previous stimulation to reflect the in vivo situation as closely as possible. Importantly, this strategy has enabled us to identify B cells infiltrating the synovial fluid and synovial tissue of RA patients as a major source of RANKL. This cytokine plays an important role in driving bone destruction in RA, and results from trials using a fully human monoclonal antibody specific for RANKL (denosumab) confirm its role in erosive progression. ${ }^{31}$

\section{METHODS}

\section{Patients}

Synovial fluid and peripheral blood were obtained from 12 patients fulfilling the 1987 American College of Rheumatology criteria for RA. ${ }^{32}$ All patients had disease of more than 3 months' duration. Synovial fluid was aspirated from joints under palpation or ultrasound guidance. Synovial tissue was taken by ultrasound-guided biopsy and 
immediately frozen in liquid nitrogen. All patient material was obtained in agreement with the Helsinki declaration. Local ethical approval was obtained and participants gave informed, written consent. Further clinical details are shown in table 1.

\section{Isolation of cell populations}

Synovial fluid was incubated with $1000 \mathrm{U} / \mathrm{ml}$ endotoxin-free hyaluronidase (Hyalase; Wockhardt, UK) at $37^{\circ} \mathrm{C}$ for $15 \mathrm{~min}$ to reduce viscosity. Mononuclear cells were isolated using density gradient centrifugation and stored at $-80^{\circ} \mathrm{C}$. Mononuclear cells were stained with mouse anti-human CD14-FITC (Immunotools, Friesoythe, Germany), CD19-PE (Immunotools), CD3-PE (eBioscience), CD45RO-FITC (Biostat, Stockport, UK), CD4-APC (eBioscience) and CD8-PE Cy7 (eBioscience) on ice. Cells were sorted into CD14, CD19, CD3 CD45RO CD4 and CD3 CD45RO CD8 populations using a MoFlo cell sorter (Beckman Coulter, High Wycombe, UK). Neutrophils were isolated from synovial fluid and peripheral blood by anti-CD15 microbeads (Miltenyi Biotec, Bisley, UK) and a magnetic-activated cell sorter. Isolated populations used had a minimum purity of $95 \%$.

\section{TaqMan low-density array analysis}

TaqMan low-density real-time PCR arrays (Applied Biosystems, Paisley, UK) were designed to determine the expression of the following genes: CCL2, CCL3, CCL4, CCL5, CCL11, CSF2, CSF3, CXCL12, EGF, FGF2, IFNA1, IFNB1, IFNG, IL1A,IL1B, IL2, IL4, IL5, IL6, IL7, IL8, IL10, IL11, IL12A, IL12B, IL13, IL15, IL17A, IL18, IL21, IL22, IL23A, IL27, LTB, MIF, TGFB1, TNFSF11(RANKL),TNFSF13, TNFSF13B, TNF, VEGFA, GAPDH and 18S. RNA was extracted using a Nucleospin XS kit (Macherey-Nagel, Düren, Germany). A reaction mixture containing RNA, Quantitect-RT Master Mix (Qiagen) and QuantiTect Reverse Transcriptase (Qiagen, Crawley, UK) was added to a TaqMan low-density array microfluidic card. Reverse transcription and real-time PCR was performed in a 7900HT Real-Time PCR System (Applied Biosystems). Relative gene expression (RO) was expressed as $2^{-\Delta \mathrm{Ct}}$ where $\Delta \mathrm{Ct}$ represents the difference in $\mathrm{Ct}$ between $18 \mathrm{~S}$ and the target gene. $\mathrm{RQ}$ values of $10^{-5}$ or less were plotted on the $\mathrm{x}$ axis.

\section{Immunofluorescence staining of synovial tissue}

Immunofluorescence staining was performed on $5 \mu \mathrm{m}$ tissue sections using mouse anti-CD3 (UCHT-1, University of Birmingham), mouse anti-CD19 FITC (Dako) and rabbit anti-
RANKL (AbCam, Cambridge, UK). CD3 was developed with goat anti-mouse Cy5 (Southern Biotech, Alabama, USA), CD19 was developed with goat anti-mouse FITC (Southern Biotech) and goat Alexa Fluor 488 anti-FITC (Molecular Probes, Paisley, UK), and RANKL was developed with donkey anti-rabbit Rhodamine (Jackson ImmunoResearch, Newmarket, UK). Concentration, and in the case of monoclonal antibodies, isotype-matched antibodies were used as controls. Sections were incubated at room temperature with primary antibody for $1 \mathrm{~h}$, washed in phosphate buffered saline (PBS), and incubated with secondary/tertiary antibodies for 30 min. PBS with $2 \%$ bovine serum albumin was used as a diluent. For nuclear counterstaining, sections were immersed in Hoechst 33258 (Sigma, Gillingham, UK) at $20 \mu \mathrm{g} / \mathrm{ml}$ for $2 \mathrm{~min}$. A Zeiss confocal LSM 510 microscope (Zeiss, Welwyn Garden City, UK) was used to capture images for processing using LSM Image Examiner software (Zeiss).

\section{Flow cytometry}

Mononuclear cells from synovial fluid and peripheral blood were stained with mouse anti-RANKL-PE (eBioscience) and CD19Pacific Blue (Biolegend, Cambridge, UK) or CD19-AlexaFluor 700 (eBioscience). PBS with $0.5 \%$ bovine serum albumin was used as a diluent and washing buffer. Flow cytometry was performed using a Cyan ADP High Performance flow cytometer (Beckman Coulter). Data were analysed using SUMMIT and FlowJo.

\section{RESULTS}

We developed a novel technical approach to systematically determine messenger RNA expression of 41 cytokines in T cells, $B$ cells, macrophages and neutrophils isolated from a single sample of synovial fluid or peripheral blood from RA patients (see supplementary figure 1, available online only). In each of the isolated cell populations, the cytokine mRNA expression profile was determined by real-time PCR. In a series of validation experiments we excluded bias due to sample preparation, normalisation and staining techniques (figure 1). The results of these preliminary experiments confirmed the robustness of the experimental approach taken and thus the validity of the results obtained in this study.

The complete set of data showing a range of cytokine expression patterns in all five cell populations in peripheral blood and synovial fluid of RA patients is shown in the supplementary data (supplementary figure 1A-D, available online only). IL-4, IL-5,

Table 1 Details on patient clinical information

\begin{tabular}{|c|c|c|c|c|c|c|c|}
\hline No & Gender (M/F) & Age, years & $\begin{array}{l}\text { Disease } \\
\text { duration, years }\end{array}$ & $\begin{array}{l}\text { Rheumatoid } \\
\text { factor }\end{array}$ & $\begin{array}{l}\text { Anti-CCP } \\
\text { antibody }\end{array}$ & DAS28 (ESR) & $\begin{array}{l}\text { Current disease-modifying } \\
\text { antirheumatic drug/ } \\
\text { glucocorticoid therapy }\end{array}$ \\
\hline 1 & $\mathrm{M}$ & 79 & 1 & Positive & Positive & 8.4 & Nil \\
\hline 2 & $\mathrm{~F}$ & 66 & 6 & Negative & Negative & 4.3 & Hydroxychloroquine \\
\hline 3 & M & 79 & 1 & Positive & Positive & 4.4 & Methotrexate, prednisolone \\
\hline 4 & $\mathrm{~F}$ & 67 & 30 & Positive & Positive & 7.1 & $\begin{array}{l}\text { Hydroxychloroquine, } \\
\text { sulphasalazine }\end{array}$ \\
\hline 5 & $\mathrm{~F}$ & 59 & 1 & Positive & Positive & 5.0 & Prednisolone \\
\hline 6 & $\mathrm{~F}$ & 73 & 18 & Positive & Unknown & 4.5 & Methotrexate \\
\hline 7 & $\mathrm{~F}$ & 58 & 6 & Positive & Positive & 5.6 & Prednisolone \\
\hline 8 & $\mathrm{~F}$ & 75 & 40 & Positive & Positive & 4.9 & Methotrexate, sulphasalazine \\
\hline 9 & $\mathrm{M}$ & 41 & 6 & Positive & Positive & 5.2 & $\begin{array}{l}\text { Methotrexate, adalimumab, } \\
\text { prednisolone }\end{array}$ \\
\hline 10 & $\mathrm{~F}$ & 55 & 5 & Positive & Positive & 7.5 & Methotrexate \\
\hline 11 & $M$ & 52 & 2 & Positive & Negative & 3.1 & Nil \\
\hline 12 & $\mathrm{M}$ & 81 & 0.5 & Positive & Positive & 6.2 & Nil \\
\hline
\end{tabular}

Anti-CCP, anti-cyclic citrullinated peptide; DAS28, disease activity score in 28 joints; ESR, erythrocyte sedimentation rate. 
A

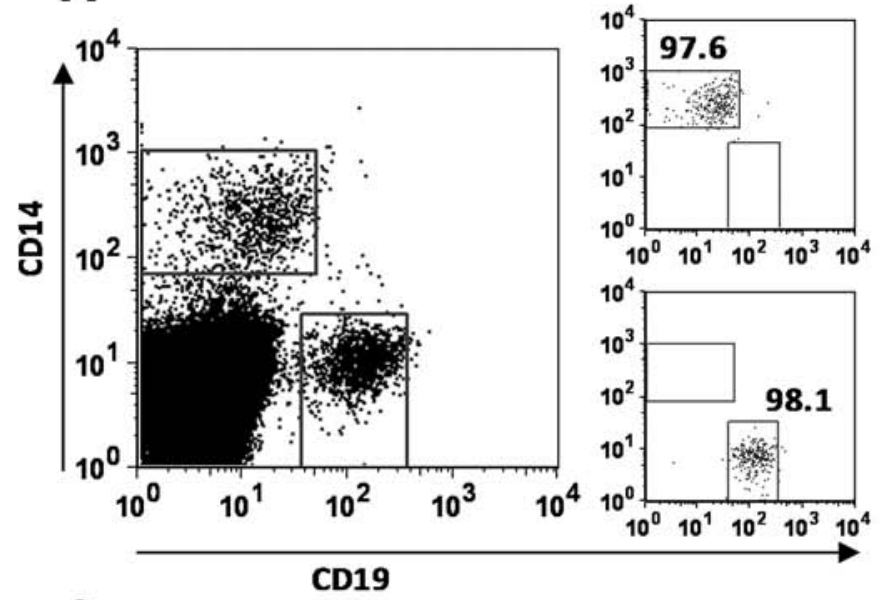

C

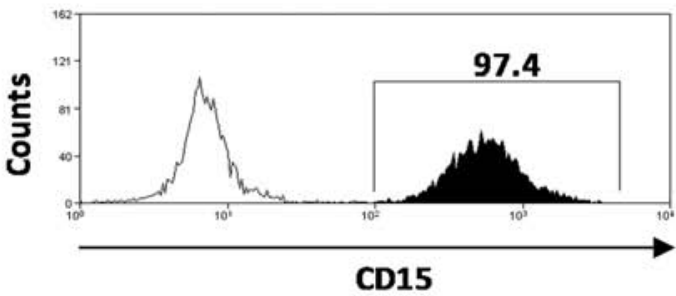

E

$\mathbf{F}$
B

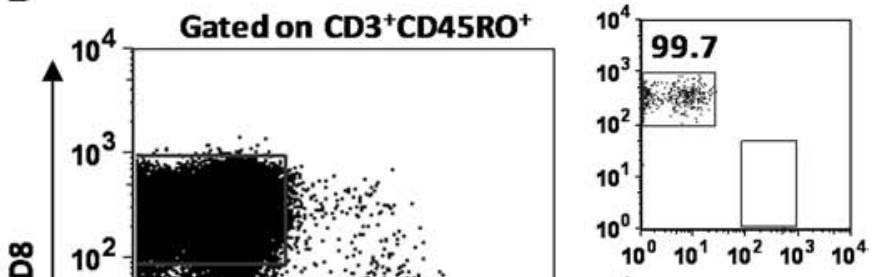

ㅇํ
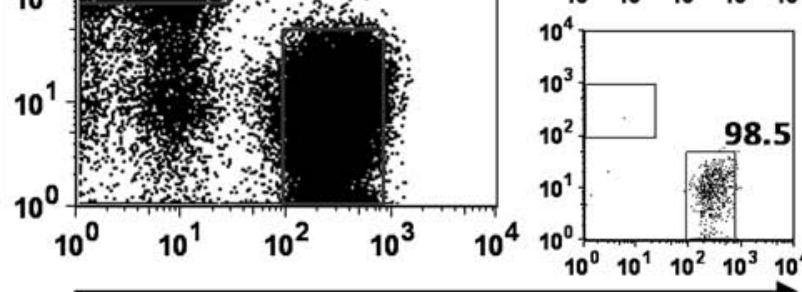

CD4

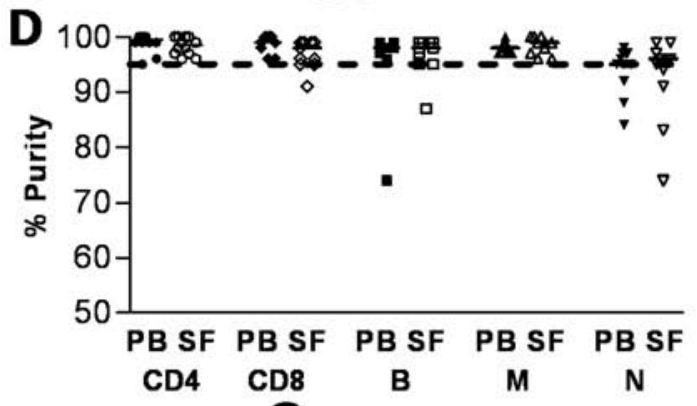

G
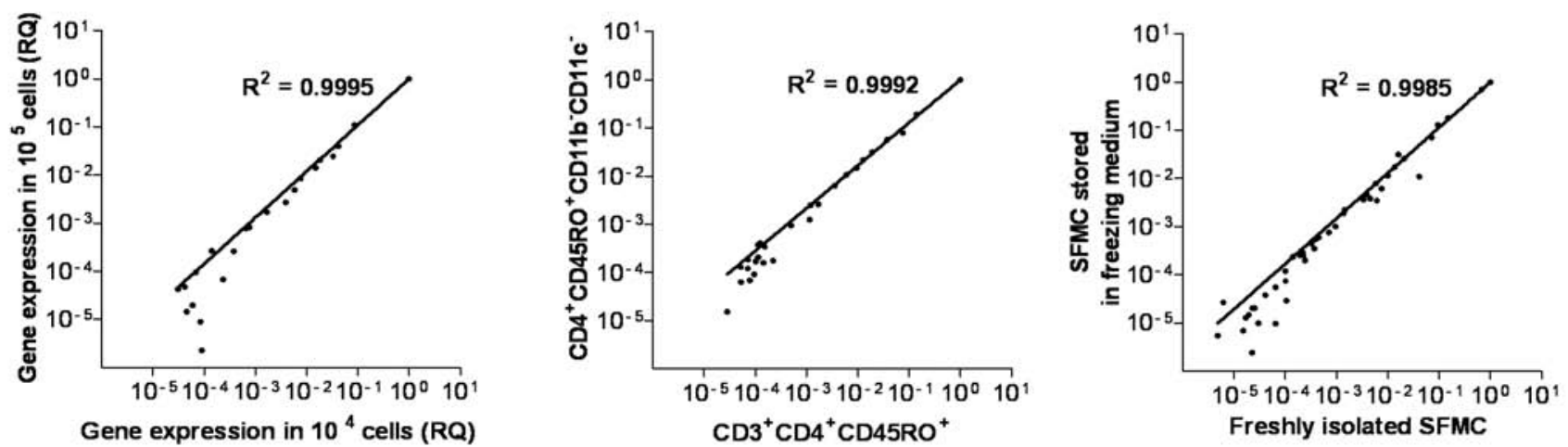

Figure 1 Method validation. (A, B) B cells, monocytes, CD4 T cells and CD8 T cells were sorted based on their expression of CD19, CD14, CD4/ $\mathrm{CD} 3 / \mathrm{CD} 45 \mathrm{R} 0$ and $\mathrm{CD} 8 / \mathrm{CD} 3 / \mathrm{CD} 45 \mathrm{R} 0$, respectively. (C) Neutrophils were isolated with CD15-coated magnetic beads. (D) All samples were re-analysed after the sort and samples with more than $5 \%$ contaminating cells were rejected. (E) To establish the cell number needed for the detection of cytokine gene expression, results for $1 \times 10^{3}, 1 \times 10^{4}$ and $1 \times 10^{5}$ cells were directly compared in three independent experiments. There was a close correlation between the results yielded by the detection of 43 individual cytokine genes in $1 \times 10^{4}$ and $1 \times 10^{5}$ cells, confirming that using a minimum cell number of $10^{4}$ would be sufficient for the sensitivity of the real-time PCR microfluidic card assay. Comparison of the results from $1 \times 10^{3}$ genes and $1 \times 10^{5}$ cells showed a large degree of variation (data not shown). (F) Comparison of cytokine gene expression for mononuclear cells sorted on the basis of $\mathrm{CD} 3$ and $\mathrm{CD} 4$ expression compared with T cells sorted on the basis of CD4 expression and lack expression of the myeloid cell markers CD11c and CD11b. Under controlled experimental conditions, keeping the cells below $4^{\circ} \mathrm{C}, \mathrm{T}$ cells sorted using anti-CD3 antibody did not have an altered expression profile compared with T cells sorted using alternative markers, showing that under these conditions there is no induction of cytokine gene expression by the CD3 antibody (data representative of three independent experiments). (G) The cytokine mRNA expression was not altered by storing synovial fluid mononuclear cells in freezing medium at $-80^{\circ} \mathrm{C}$ compared with using freshly isolated cells (data representative of two independent experiments). PB, peripheral blood; SF, synovial fluid; SFMC, synovial fluid mononuclear cell.

IL-13, IL-22, CCL11, CXCL12, CSF-3 and EGF mRNA was undetectable in the majority of patients (data not shown). Positive controls (mRNA from $T$ cells stimulated with magnetic beads coated with anti-CD3 and anti-CD28 antibody and a commercially available blend of RNA from human cell lines representing different tissues (Stratagene, Stockport, UK)) were used to test whether these assays worked. With the exception of CSF-3 all of the cytokine mRNA species were detected.
A selection of cytokines expressed predominantly by lymphocytes in the synovial fluid is shown in figure 1. In synovial fluid $T$ cells we detected the expression of IFN $\gamma$ and IL-2 mRNA. In the CD4 cell population we observed the expression of IL-17A mRNA suggesting the presence of T-helper (Th) type 17 cells. We also found that IL-21, a cytokine reportedly produced by Th17 cells, was expressed in the CD4 T-cell population. ${ }^{33}$ The cytokines IL-12 and IL-23 are important regulators 
of differentiation and survival of Th1 and Th17 cells, respectively. Both cytokines are produced as heterodimers and have a common p40 subunit. The synovial fluid B-cell population expressed high levels of IL-12p40 as well as IL-12p35 mRNA, suggesting that these cells may produce functionally active IL-12 (figure 2). In agreement with previous publications, $\mathrm{T}$ cells expressed mRNA coding for the IL-23p19 subunit, which combines with the IL-12p40 subunit. ${ }^{34}$ However, T cells did not express IL-12p40 mRNA, again leaving B cells as the only cell population among those analysed here expressing mRNA for both subunits required for the production of IL-23. High levels of mRNA-expression for CSF-2 (granulocyte macrophage colony-stimulating factor) by $\mathrm{CD} 4 \mathrm{~T}$ cells suggests a role for these cells in neutrophil survival. LT- $\beta$ and IL-7 mRNA were both expressed in $\mathrm{T}$ cells and $\mathrm{B}$ cells, indicating a contribution of both cell types to B-cell development and T-cell survival.

Intriguingly, RANKL (TNFSF11) mRNA was expressed predominantly by synovial fluid B cells. Previous studies have reported the expression of RANKL by RA synovial fibroblasts, $T$ cells and chondrocytes, but this is the first report of RANKL expression in B cells in RA. We also found RANKL gene expression in CD4 T cells, but at a lower level compared with B cells. The expression of RANKL at the protein level is described in a later section of this paper and in figure 5 .

The data shown in figure 3 describe cytokine mRNA expression found predominantly in the synovial fluid myeloid cell populations investigated (neutrophils and macrophages). Our results indicate that macrophages constitute the major source of the pro-inflammatory cytokine IL-1 $\beta$, the Th1-differentiation factor IL-18 and IL-27, which is implicated in the differentiation of several T-cell subsets. Macrophages were also found to be the main source of APRIL, CCL2, CCL3 and VEGF- $\alpha$ mRNA expression in RA synovial fluid, confirming their role in directing immune cell migration and differentiation as well as angiogenesis. Synovial fluid neutrophils were found to express a range of cytokines very similar to macrophages. Particularly prominent was their expression of IFN $\alpha 1$, CXCL8 and BAFF mRNA.
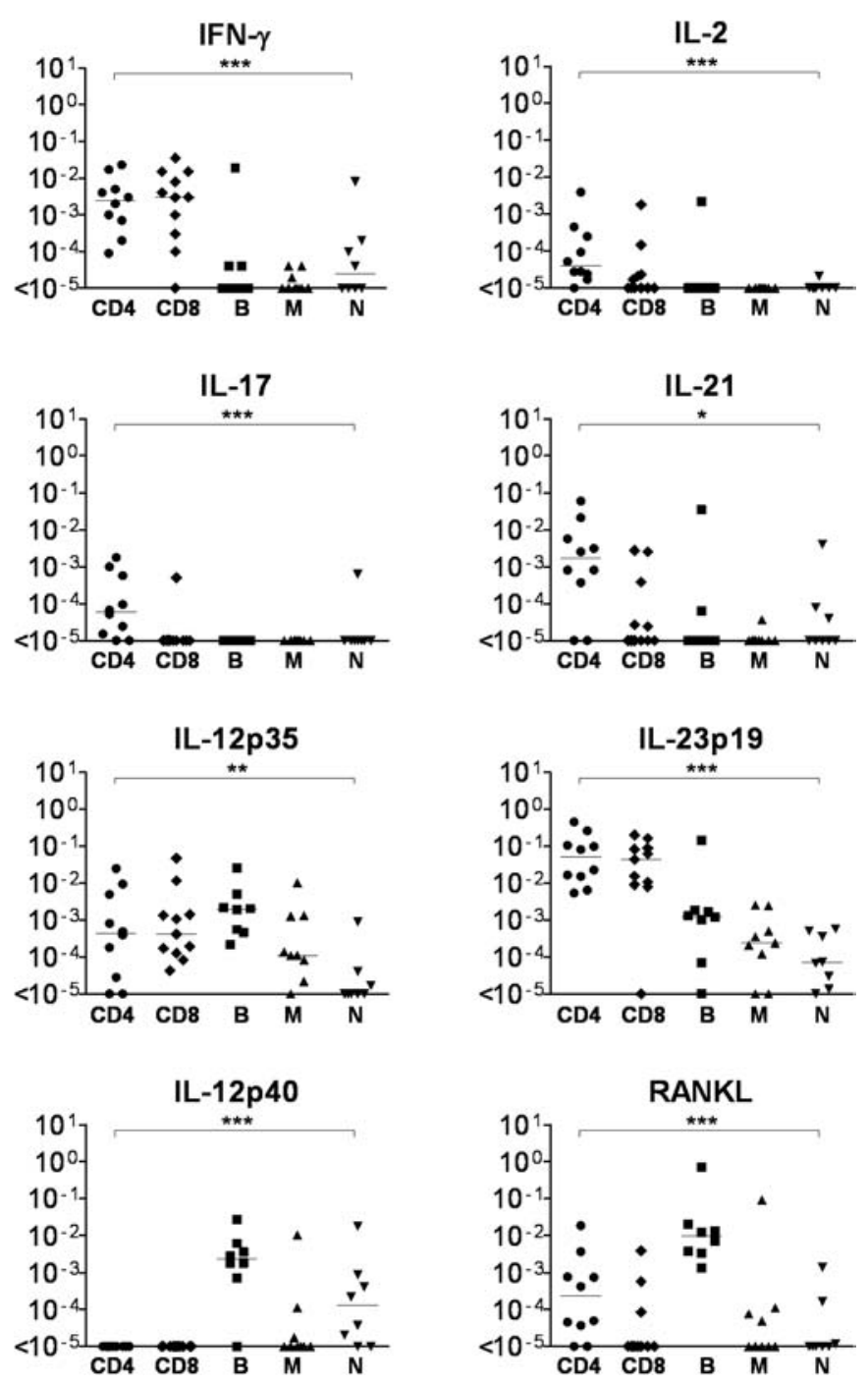

Figure 2 Cytokine genes expressed predominantly in synovial fluid lymphoid cells. Shown are the quantitative PCR results for the mRNA expression for a selection of the cytokines predominantly expressed in CD4 T cells, CD8 T cells or in CD19 B cells (B). Statistical significance was assessed by the Kruskal-Wallis test. ${ }^{*} p<0.05,{ }^{*} p<0.001$, $* * * p<0.0001$.
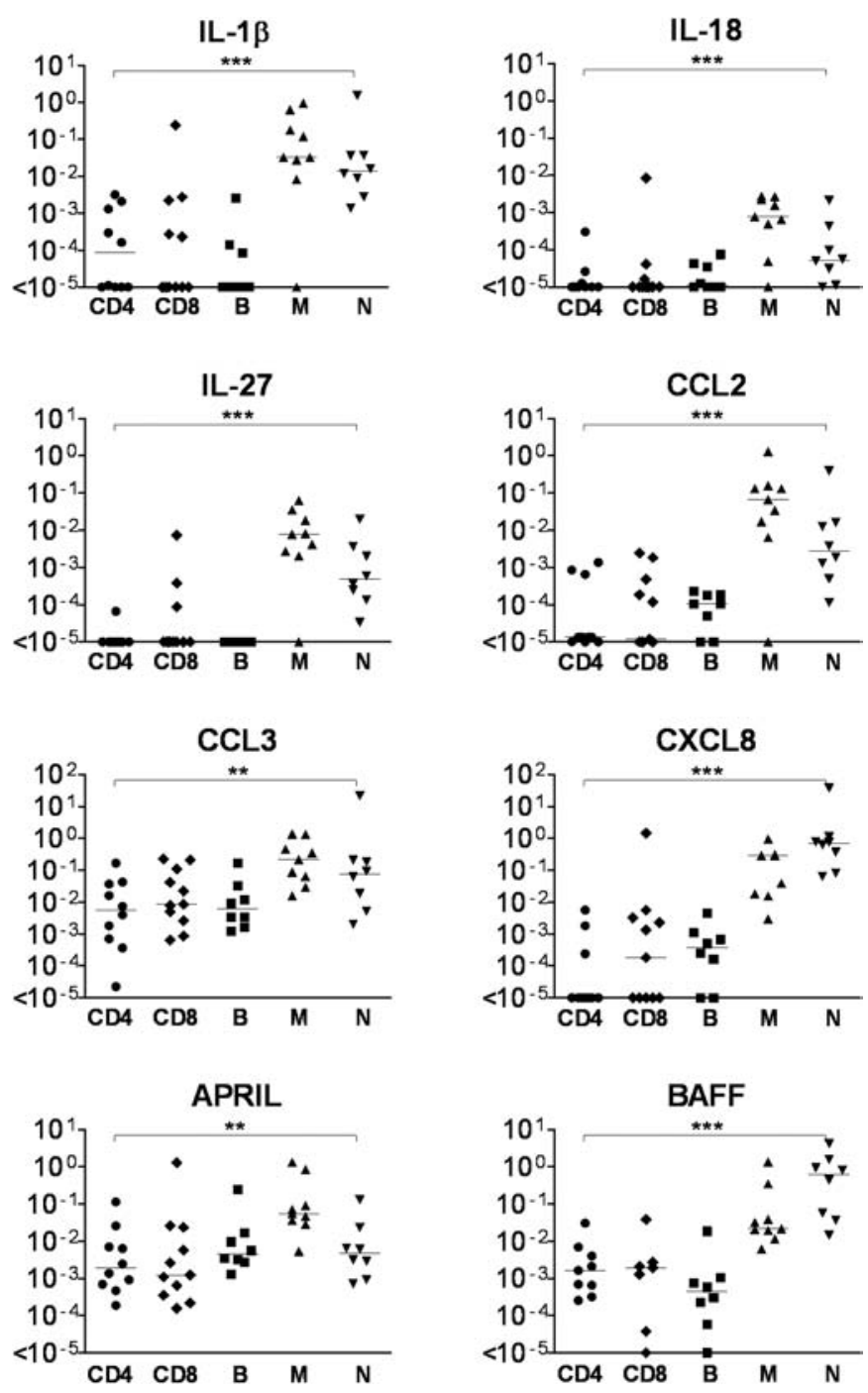

Figure 3 Cytokine genes expressed predominantly in synovial fluid macrophages or neutrophils. Shown are the quantitative PCR results for the mRNA expression for a selection of the cytokines (N) predominantly expressed in either CD14 monocytes (M) or CD15 neutrophils.

Statistical significance was assessed by the Kruskal-Wallis test. ${ }^{* *} \mathrm{p}<0.001,{ }^{* * *} \mathrm{p}<0.0001$. 
Cytokines highly expressed in both lymphocyte and myeloid cell populations are shown in figure 4 . IL-1 $\alpha$ was expressed at similar levels by macrophages, B cells and neutrophils in RA synovial fluid. B cells and macrophages comprised the major sources of IL-6, while T cells and macrophages were the primary producers of IL-10 mRNA. T-cell, B-cell and macrophage populations all expressed IL-15. TNF $\alpha$, TGF $\beta 1$, MIF and CCL4 mRNA were highly expressed by all lymphocyte and myeloid cell populations investigated.

Comparison of cytokine profiles in all five cell populations in synovial fluid and peripheral blood showed that a wide range of cytokines was expressed at a higher level in synovial fluid (see supplementary figure 3 , available online only). In the T-cell, B-cell and macrophage populations the majority of cytokines detected were over-expressed at the site of inflammation, in the synovial fluid. Interestingly, this was not the case for neutrophils.

Several novel observations of cytokine gene expression have been made in the context of this study. Because of its clinical relevance to the progression of joint destruction in RA patients, the observation of RANKL mRNA expression in synovial fluid $\mathrm{B}$ cells was extended to the protein level. First, the mRNA gene expression data were normalised to cell number (figure 5A) in order to exclude potential bias arising from normalisation to $18 \mathrm{~S}$ rRNA. Confirming our original observation, PCR data normalised to cell number identified synovial fluid CD19 B cells as the dominant producers of RANKL mRNA, while a smaller proportion of CD4 $\mathrm{T}$ cells also showed RANKL mRNA expression. The surface expression of RANKL on synovial fluid mononuclear cells was determined by co-labelling for CD19 and RANKL and flow cytometric analysis. While only a very small proportion of $B$ cells expressed RANKL in the peripheral blood of RA patients, a significantly higher proportion of $B$ cells from synovial fluid samples expressed RANKL on their surface (figure 5).

A large proportion of the B cells in the inflamed joint are localised in the synovial tissue, while they are a relatively small population among the synovial fluid cells (see supplementary figure 2, available online only). To test whether synovial tissue $B$ cells produced RANKL, frozen sections of synovial tissue from biopsies taken from RA patients with active disease were stained with antibodies to RANKL and CD19. Distinct RANKL expression was identified in CD19+ B cells in the inflamed synovium (figure 5). This observation was mainly made in B cells dispersed throughout the sublining and more rarely in lymphoid aggregates.

\section{DISCUSSION}

In this study a novel strategy was used to compare cytokine expression in the five dominant immune cell populations in the synovial fluid and peripheral blood of RA patients without ex vivo stimulation. We also detected the expression of several chemokines and growth factors that contribute to cell recruitment and survival in the RA joint. ${ }^{35}$ While many cytokines were found in the populations we would have predicted based on the existing literature, we also made several unexpected findings. Notably, we observed that synovial fluid B cells express high levels of pro-inflammatory cytokines. The B-cell population was the only synovial fluid population that expressed both IL-12p35 and IL-12p 40 , the two subunits that are required for the production of functional IL-12. This observation is supported by previous reports of IL-12 production by B cells. ${ }^{36}$ Synovial fluid B cells also expressed IL-23p19, the subunit that heterodimerises with IL-12p40 to form IL-23, indicating that synovial fluid B cells also have the capacity to produce functional IL-23. We found that
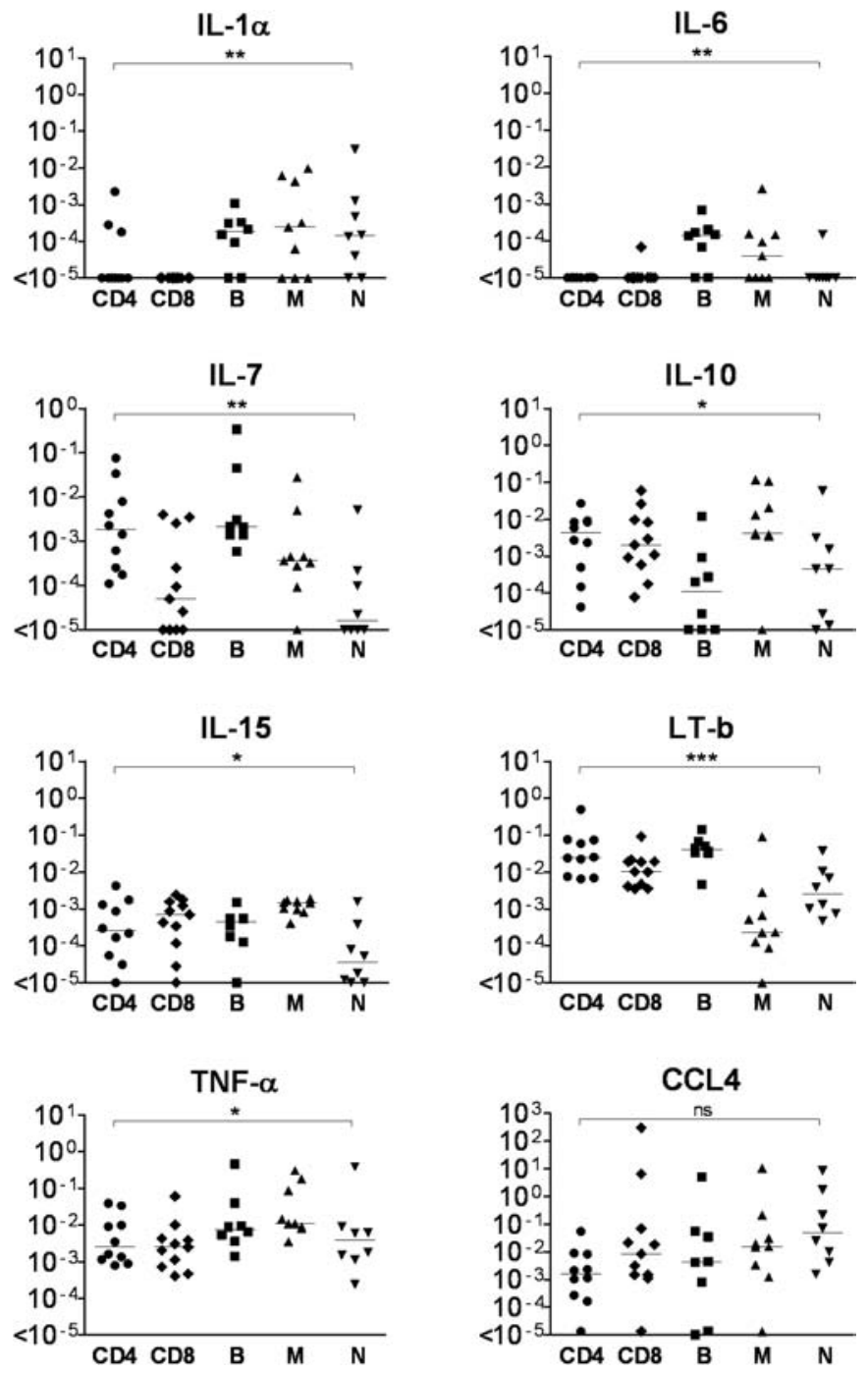

Figure 4 Cytokines expressed in both synovial fluid myeloid and lymphoid cell populations. Shown are the quantitative PCR results for the mRNA expression for a selection of the cytokines expressed in at least one of the lymphoid cell populations: CD4 T cells, CD8 T cells or in CD19 B cells as well as either CD14 monocytes or CD15 neutrophils. Statistical significance was assessed by the Kruskal-Wallis test. ${ }^{*} p<0.05,{ }^{* *} p<0.001,{ }^{* * *} p<0.0001$.

IL-23p19 was expressed predominantly by synovial fluid T cells, in agreement with the original publication that reported high levels of IL-23p19 in polarised Th1 cells. ${ }^{34}$ However, our observation that synovial fluid CD4 and CD8 $\mathrm{T}$ cells do not express IL-12p40 mRNA raises the question of whether synovial fluid $\mathrm{T}$ cells express a yet undescribed alternative binding partner for IL-23p19. A discrepancy between the level of IL-23p19 mRNA expression and functional expression of IL-23 at the protein level has also been described in RA synovial tissue and fibroblast-like synoviocytes. ${ }^{37}$

While it is thought that differentiation towards plasma cells and the production of rheumatoid factor and anti-citrullinated protein antibodies contribute to disease, B cells are likely to play further roles. Antigen presentation by B cells is likely to play a role in the local activation of $\mathrm{T}$ cells thus indirectly increasing T-cell cytokine production, but little is known about direct cytokine production by B cells in the RA synovium. ${ }^{38}$ An antiinflammatory role has been proposed for a subset of $B$ cells that can produce IL-10.18 193940 These cells have been observed in 



D



Figure 5 Expression of receptor activator nuclear factor kappa B ligand (RANKL) in B cells infiltrating the rheumatoid synovial tissue and fluid. (A) Quantitative PCR data for detection of RANKL in sorted peripheral blood (PB) and synovial fluid (SF) CD4 and CD8 T cells, CD19 B cells and CD14 monocytes/macrophages. Data normalised to the exact number of cells sorted. (B) Flow cytometric detection of RANKL. Peripheral blood and synovial fluid mononuclear cells were co-labelled with anti-CD19 and either isotype matched control or anti-RANKL antibody. Staining representative of samples from eight patients with rheumatoid arthritis (RA). (C) Percentage of RANKL ${ }^{+}$cells among the CD19 B cells of RA patients as detected by flow cytometry. (D) Detection of RANKL ${ }^{+}$B cells in synovial tissue. Synovial tissue was stained for CD19 (green), and RANKL (red) and viewed at a final magnification of $\times 400$. Representative sections from five RA patients were investigated.

peripheral blood of patients with autoimmune diseases including RA, but have not been reported in the rheumatoid joint so far. The mRNA cytokine profile of synovial fluid B cells detected in this study does not support a major contribution of B cells to IL-10 production, but rather supports a generally proinflammatory role for this cell type in the synovial fluid.

This is the first study to report that B cells contribute to RANKL production in the inflamed rheumatoid joint. Previous studies have reported the expression of RANKL by RA synovial fibroblasts, T cells and chondrocytes, and that levels of soluble RANKL are elevated in synovial fluid compared with the serum of RA patients. ${ }^{41-44}$ RANKL is known to be required for osteoclast development and plays a key role in mediating bone erosion. ${ }^{45}$ Of relevance to our observation that synovial B cells express this cytokine, RANKL has been identified as part of a distinct expression profile that defines a subset of memory B cells that normally reside in epithelial niches. ${ }^{46}$ Furthermore, RANKL has been detected in B cells in the gingival tissue of patients with periodontal disease and in plasma cells in osteolytic myeloma bone disease, in which it is associated with bone degradation. ${ }^{47} 48$

RANKL-deficient mice have altered $B$ and $T$ cell populations and lack lymph nodes indicating that RANKL plays a role in lymphocyte development and lymph node organogenesis. ${ }^{45}$ The development of ectopic lymphoid structures and lymphoid neogenesis is a poorly understood feature of many chronic inflammatory diseases including RA. ${ }^{49}$ It will be intriguing to investigate how the production of RANKL by B cells at sites of chronic inflammation contributes to the development of these lymphoid aggregates.

Data from the REFLEX trial revealed that the B-cell targeting therapy rituximab, in combination with methotrexate, caused significant reduction in joint damage progression compared with methotrexate alone. ${ }^{50}$ Together with our observation of RANKL production by $B$ cells in the synovial tissue and fluid of RA 
patients, this suggests that depletion of RANKL-expressing B cells may contribute to the inhibition of bone erosion by rituximab. ${ }^{50}$

Acknowledgements The authors gratefully acknowledge Mr Roger Bird and Miss Katherine Howlett for expert technical assistance.

Funding This work was funded by an Arthritis Research UK career progression fellowship to DS-T (19394), a programme grant from Arthritis Research UK to MS and CDB (16390), two Arthritis Research UK equipment grants to DS-T and KR (17767, 18198), an MRC-funded PhD studentship to LY and the EU FP6-funded consortium 'Autocure'.

\section{Competing interests None.}

Patient consent Obtained.

Ethics approval This study was conducted with the approval of the Solihull Local Research Ethics Committee.

Provenance and peer review Not commissioned; externally peer reviewed

\section{REFERENCES}

1. Brennan FM, Mclnnes IB. Evidence that cytokines play a role in rheumatoid arthritis. J Clin Invest 2008:118:3537-45.

2. Feldmann $\mathbf{M}$, Maini SR. Role of cytokines in rheumatoid arthritis: an education in pathophysiology and therapeutics. Immunol Rev 2008;223:7-19.

3. van den Berg WB. Lessons from animal models of arthritis over the past decade. Arthritis Res Ther 2009:11:250.

4. Ehrenstein MR, Mauri C. If the treatment works, do we need to know why?: the promise of immunotherapy for experimental medicine. J Exp Med 2007;204:2249-52.

5. Brennan FM, Chantry D, Jackson A, et al. Inhibitory effect of TNF alpha antibodies on synovial cell interleukin-1 production in rheumatoid arthritis. Lancet 1989;2:244-7.

6. McInnes IB, Schett G. Cytokines in the pathogenesis of rheumatoid arthritis Nat Rev Immunol 2007:7:429-42.

7. Combe B, Pope RM, Fischbach M, et al. Interleukin-2 in rheumatoid arthritis: production of and response to interleukin-2 in rheumatoid synovial fluid, synovia tissue and peripheral blood. Clin Exp Immunol 1985;59:520-8.

8. Firestein GS, Xu WD, Townsend $\mathrm{K}$, et al. Cytokines in chronic inflammatory arthritis. I. Failure to detect $T$ cell lymphokines (interleukin 2 and interleukin 3 ) and presence of macrophage colony-stimulating factor (CSF-1) and a novel mast cell growth factor in rheumatoid synovitis. J Exp Med 1988;168:1573-86.

9. Smeets TJ, Dolhain RJEM ,AM, et al. Poor expression of T cell-derived cytokines and activation and proliferation markers in early rheumatoid synovial tissue. Clin Immunol Immunopathol 1998;88:84-90.

10. Chabaud M, Durand JM, Buchs N, et al. Human interleukin-17: a T cell-derived proinflammatory cytokine produced by the rheumatoid synovium. Arthritis Rheum 1999:42:963-70.

11. Kotake S, Udagawa N, Takahashi N, et al. IL-17 in synovial fluids from patients with rheumatoid arthritis is a potent stimulator of osteoclastogenesis. J Clin Invest 1999;103:1345-52

12. Field $\mathbf{M}$, Chu $\mathbf{C}$, Feldmann $\mathbf{M}$, et al. Interleukin-6 localisation in the synovial membrane in rheumatoid arthritis. Rheumatol Int 1991;11:45-50.

13. Chu Co, Field M, Feldmann $\mathrm{M}$, et al. Localization of tumor necrosis factor alpha in synovial tissues and at the cartilage-pannus junction in patients with rheumatoid arthritis. Arthritis Rheum 1991;34:1125-32.

14. Crotti TN, Smith MD, Weedon H, et al. Receptor activator NF-kappaB ligand (RANKL) expression in synovial tissue from patients with rheumatoid arthritis, spondyloarthropathy, osteoarthritis, and from normal patients: semiquantitative and quantitative analysis. Ann Rheum Dis 2002;61:1047-54.

15. Takemura $\mathbf{S}$, Braun A, Crowson C, et al. Lymphoid neogenesis in rheumatoid synovitis. J Immunol 2001;167:1072-80.

16. Weyand CM, Seyler TM, Goronzy JJ. B cells in rheumatoid synovitis. Arthritis Res Ther 2005; 7 (Suppl 3):S9-12

17. Hirano T, Matsuda T, Turner M, et al. Excessive production of interleukin 6/B cell stimulatory factor-2 in rheumatoid arthritis. Eur J Immunol 1988;18:1797-801.

18. Llorente L, Richaud-Patin Y, Fior R, et al. In vivo production of interleukin-10 by non-T cells in rheumatoid arthritis, Sjögren's syndrome, and systemic lupus erythematosus. A potential mechanism of B lymphocyte hyperactivity and autoimmunity. Arthritis Rheum 1994;37:1647-55.

19. Fillatreau S, Sweenie $\mathrm{CH}, \mathrm{McGeachy}$ MJ, et al. B cells regulate autoimmunity by provision of IL-10. Nat Immunol 2002;3:944-50.

20. Wood NC, Dickens E, Symons JA, et al. In situ hybridization of interleukin-1 in CD14positive cells in rheumatoid arthritis. Clin Immunol Immunopathol 1992;62:295-300.

21. Tanaka M, Harigai M, Kawaguchi $Y$, et al. Mature form of interleukin 18 is expressed in rheumatoid arthritis synovial tissue and contributes to interferon-gamma production by synovial T cells. J Rheumatol 2001:28:1779-87.

22. van Roon JA, Hartgring SA, Wenting-van Wijk M, et al. Persistence of interleukin 7 activity and levels on tumour necrosis factor alpha blockade in patients with rheumatoid arthritis. Ann Rheum Dis 2007;66:664-9.
23. van Holten J, Smeets TJ, Blankert P, et al. Expression of interferon beta in synovial tissue from patients with rheumatoid arthritis: comparison with patients with osteoarthritis and reactive arthritis. Ann Rheum Dis 2005:64:1780-2.

24. Stamp LK, Easson A, Pettersson L, et al. Monocyte derived interleukin (IL)-23 is an important determinant of synovial IL-17A expression in rheumatoid arthritis. $J$ Rheumatol 2009;36:2403-8.

25. Gabay C, Krenn V, Bosshard C, et al. Synovial tissues concentrate secreted APRIL. Arthritis Res Ther 2009;11:R144.

26. Seyler TM, Park YW, Takemura S, et al. BLyS and APRIL in rheumatoid arthritis. $J$ Clin Invest 2005;115:3083-92.

27. Beaulieu AD, McColl SR. Differential expression of two major cytokines produced by neutrophils, interleukin-8 and the interleukin-1 receptor antagonist, in neutrophils isolated from the synovial fluid and peripheral blood of patients with rheumatoid arthritis. Arthritis Rheum 1994:37:855-9.

28. Assi LK, Wong SH, Ludwig A, et al. Tumor necrosis factor alpha activates release of $B$ lymphocyte stimulator by neutrophils infiltrating the rheumatoid joint. Arthritis Rheum 2007;56:1776-86.

29. Quayle JA, Adams S, Bucknall RC, et al. Cytokine expression by inflammatory neutrophils. FEMS Immunol Med Microbiol 1994:8:233-9.

30. Hatano $\mathbf{Y}$, Kasama T, Iwabuchi $\mathrm{H}$, et al. Macrophage inflammatory protein 1 alpha expression by synovial fluid neutrophils in rheumatoid arthritis. Ann Rheum Dis 1999;58:297-302.

31. Cohen SB, Dore RK, Lane NE, et al. Denosumab treatment effects on structural damage, bone mineral density, and bone turnover in rheumatoid arthritis: a twelve month, multicenter, randomized, double-blind, placebo-controlled, phase II clinical trial. Arthritis Rheum 2008;58:1299-309.

32. Arnett FC, Edworthy SM, Bloch DA, et al. The American Rheumatism Association 1987 revised criteria for the classification of rheumatoid arthritis. Arthritis Rheum 1988;31:315-24

33. Wei L, Laurence A, Elias KM, et al. IL-21 is produced by Th17 cells and drives IL-17 production in a STAT3-dependent manner. J Biol Chem 2007;282:34605-10.

34. Oppmann B, Lesley R, Blom B, et al. Novel p19 protein engages IL-12p40 to form a cytokine, IL-23, with biological activities similar as well as distinct from IL-12. Immunity 2000;13:715-25.

35. Salmon M, Scheel-Toellner D, Huissoon AP, et al. Inhibition of T cell apoptosis in the rheumatoid synovium. J Clin Invest 1997:99:439-46.

36. Airoldi I, Guglielmino R, Carra G, et al. The interleukin-12 and interleukin-12 receptor system in normal and transformed human B lymphocytes. Haematologica 2002:87:434-42

37. Brentano F, Ospelt C, Stanczyk J, et al. Abundant expression of the interleukin (IL)23 subunit p19, but low levels of bioactive IL23 in the rheumatoid synovium: differential expression and Toll-like receptor-(TLR) dependent regulation of the IL23 subunits, p19 and p40, in rheumatoid arthritis. Ann Rheum Dis 2009;68:143-50.

38. Takemura $\mathbf{S}$, Klimiuk PA, Braun A, et al. T cell activation in rheumatoid synovium is B cell dependent. J Immunol 2001:167:4710-18.

39. Evans JG, Chavez-Rueda KA, Eddaoudi A, et al. Novel suppressive function of transitional 2 B cells in experimental arthritis. J Immunol 2007;178:7868-78.

40. Mauri C, Gray D, Mushtaq N, et al. Prevention of arthritis by interleukin 10-producing B cells. J Exp Med 2003;197:489-501.

41. Shigeyama Y, Pap T, Kunzler P, et al. Expression of osteoclast differentiation factor in rheumatoid arthritis. Arthritis Rheum 2000;43:2523-30

42. Skoumal M, Kolarz G, Haberhauer G, et al. Osteoprotegerin and the receptor activator of NF-kappa B ligand in the serum and synovial fluid. A comparison of patients with longstanding rheumatoid arthritis and osteoarthritis. Rheumatol Int 2005;26:63-9.

43. Ziolkowska M, Kurowska M, Radzikowska A, et al. High levels of osteoprotegerin and soluble receptor activator of nuclear factor kappa B ligand in serum of rheumatoid arthritis patients and their normalization after anti-tumor necrosis factor alpha treatment. Arthritis Rheum 2002;46:1744-53.

44. Gravallese EM, Manning C, Tsay A, et al. Synovial tissue in rheumatoid arthritis is a source of osteoclast differentiation factor. Arthritis Rheum 2000:43:250-8.

45. Kong YY, Yoshida H, Sarosi I, et al. OPGL is a key regulator of osteoclastogenesis lymphocyte development and lymph-node organogenesis. Nature 1999;397:315-23.

46. Ehrhardt GR, Hijikata A, Kitamura $\mathrm{H}$, et al. Discriminating gene expression profiles of memory B cell subpopulations. J Exp Med 2008;205:1807-17.

47. Kawai T, Matsuyama T, Hosokawa Y, et al. B and T lymphocytes are the primary sources of RANKL in the bone resorptive lesion of periodontal disease. Am J Pathol 2006:169:987-98.

48. Heider U, Langelotz C, Jakob C, et al. Expression of receptor activator of nuclear factor kappaB ligand on bone marrow plasma cells correlates with osteolytic bone disease in patients with multiple myeloma. Clin Cancer Res 2003:9:1436-40.

49. Manzo A, Bombardieri M, Humby F, et al. Secondary and ectopic lymphoid tissue responses in rheumatoid arthritis: from inflammation to autoimmunity and tissue damage/remodeling. Immunol Rev 2010;233:267-85.

50. Keystone E, Emery P, Peterfy CG, et al. Rituximab inhibits structural joint damage in patients with rheumatoid arthritis with an inadequate response to tumour necrosis factor inhibitor therapies. Ann Rheum Dis 2009:68:216-21. 